\title{
THE INFLUENCE OF ELASTICITY ON ANALYSIS: THE CLASSIC HERITAGE
}

\author{
BY C. TRUESDELL \\ Dedicated to J. L. Ericksen on his 60th birthday
}

\section{Contents}

1. The catenary: twin solutions, and the calculus of variations.

2. The elastica: elliptic functions, qualitative analysis, multiple solutions, differential geometry of skew curves.

3. Systems in small vibration: proper numbers and simple modes, linear differential equations, "Bessel” functions, "Laguerre" polynomials, integral transforms, resonant oscillations.

4. Partial differential equations: the concept of "solution", discontinuities, the "modern" definition of function, functional basis, singularities, generalized solutions.

5. Plates: the "Gaussian" curvature.

6. The three-dimensional theory: deformation of regions, strain and local rotation, tensors, proper numbers and proper vectors, polar decomposition, spectral resolution, isotropy and hemitropy, groups.

7. Torsion of a cylinder: principal solution of a system of differential equations and its status as an approximation to other solutions.

Most mathematicians have an idea of the influence of hydrodynamics and electromagnetism on the theory of complex functions and harmonic potentials. The influence of elasticity is less well known. Elasticity led to a vast range of mathematical problems involving linear algebra, differential geometry, ordinary and partial differential equations (mostly nonlinear), elliptic functions, and the calculus of variations.

1. The catenary: twin solutions, and the calculus of variations. The contest of 1690 to find the catenary curve is described in all histories of mathematics. The body treated is a chain or rope without stiffness. The mathematical model for this body is a plane curve. This curve satisfies differential equations expressing the requirement that the resultant force and torque on each part of the body be zero. As early as 1675 Hooke had stated in an anagram, "as hangs the flexible line, so but inverted will stand the rigid arch", but Hooke was not a mathematician and could not prove anything. His statement applies nevertheless to Leibniz's differential equation for the catenary: If that equation has a solution bellied downward for the points considered, it also has one bellied upward

This article is based on a lecture given at the AMS-MAA joint symposium, Toronto, Canada on August 25, 1982; received by the editors May 26, 1983.

1980 Mathematics Subject Classification. Primary 73-02; Secondary 15-02, 53-02, 33-02, 33A25, 49-02. 
(Figure 1). Thus in the very infancy of differential equations we encounter a boundary-value problem that fails to have a unique solution. Each of the two solutions of the differential equation is the unique solution of a variational principle: For one, the center of gravity hangs as low as possible; for the other, it rises as high as possible. The catenary provides one of the "isoperimetric" problems that led James Bernoulli to invent the calculus of variations in the immediately following years.

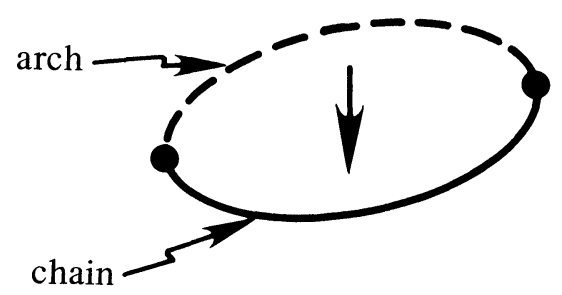

FIGURE 1. Hanging chain and ideal arch, both solutions of the differential equation for the catenary through two given points.

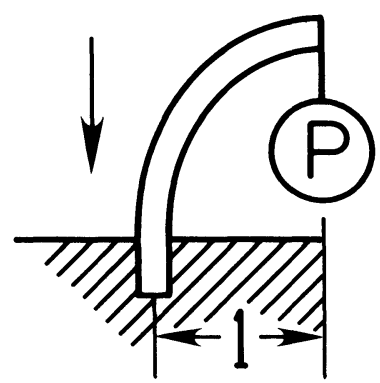

FIGURE 2. Rectangular elastica of unit excursion

2. The elastica: elliptic functions, qualitative analysis, multiple solutions, differential geometry of skew curves. In 1691 James Bernoulli proposed the problem of the bent beam, elastic bar, or simply "elastica" (Figure 2). A good example of an elastica in real life, at least of yesteryear, is a corset stay. Bernoulli's problem was twofold: first derive the governing equations, then solve them. In mathematical practice today it is, unfortunately, often forgotten that to derive basic equations is even so much a mathematician's duty as to study their properties. Bernoulli concealed his solution of the twofold problem in an anagram, as Leibniz had his for the catenary. He explained that to solve the problem he needed first to discover and prove a "golden theorem"; this theorem provides the curvature of a plane curve. Although Huygens had already published an equivalent statement in his book on the pendulum clock, it was obscurely expressed, and we may adduce James Bernoulli's golden rediscovery as an early instance of the influence of elasticity upon differential 
geometry. There is no accident here. Bernoulli's simplest hypothesis makes the moment needed to bend an initially straight bar inversely proportional to the radius of curvature produced:

$$
\text { Bending moment }=\frac{\mathscr{B}}{r} \quad\left\{\begin{array}{l}
\mathscr{B}=\text { "flexural rigidity" of bar, } \\
r=\text { radius of curvature of bent bar. }
\end{array}\right.
$$

No theory based upon this hypothesis could be produced except by a man who disposed of a way to get $r$. While the problem of the catenary had been solved almost at once, not only by Leibniz but also by Huygens and by James Bernoulli's younger brother John, this second problem is deeper, and James Bernoulli had the triumphant consolation of receiving not a single answer in three years, whereupon he published his solution. For an elastic bar (Figure 2) of unit excursion, built vertically into a horizontal wall and bent by a load sufficient to make its top horizontal-the instance called the rectangular elastica-the differential system is

$$
d y=\frac{x^{2} d x}{\sqrt{1-x^{4}}}, \quad d s=\frac{d x}{\sqrt{1-x^{4}}}, \quad 0 \leqslant x \leqslant 1 .
$$

You will recognize the second quadrature as making the excursion $x$ a lemniscate function of the arc length $s$; this function was to reappear in the work of Fagnano twenty-one years later. Bernoulli integrated his equation in series and calculated precise upper and lower bounds for their values at $x=1$. He wrote, "I have heavy grounds to believe that the construction of our curve depends neither on the quadrature nor on the rectification of any conic section." Thus the elliptic functions were born.

The problem of the rectangular elastica joined that of the catenary in providing one of the impulses to James Bernoulli's calculus of variations. In 1695 he wrote, "Among all the curves of given length drawn over the same straight line the elastica is the one such that the center of gravity of the included area is farthest from the line...." Some forty-three years later his nephew Daniel Bernoulli suggested to Euler that all kinds of elastica should satisfy the variational principle

$$
\int \frac{d s}{r^{2}}=\text { minimum. }
$$

Five years after that, in 1743 , when Euler was writing his great treatise on the calculus of variations, Bernoulli repeated the suggestion. Euler seized the occasion to adjoin to his book an appendix in which he took a few lines to derive from Daniel Bernoulli's principle the differential system

$$
d y=\frac{\left(\alpha+\beta x+\gamma x^{2}\right) d x}{\sqrt{a^{4}-\left(\alpha+\beta x+\gamma x^{2}\right)^{2}}}, \quad d s=\frac{a^{2} d x}{\sqrt{a^{4}-\left(\alpha+\beta x+\gamma x^{2}\right)^{2}}},
$$

in which $\alpha / a^{2}, \beta / a$, and $\gamma$ are real parameters expressible in terms of $\mathscr{B}$, the load, and the prescribed length. The rest of Euler's appendix is a treatise on the equilibria of bent rods and the small vibrations of nearly straight rods. In 
solving the former group of problems, Euler determines precisely all forms the elastic curve may take. The analysis is more difficult than for the catenary because the results depend upon the arbitrary material parameter $\Re$. The catenary and the ideal fluid are degenerate examples of mechanical systems in that they are only trivially affected by material properties. The elastica provided the first instance of the analytic complications that dependence upon an essential parameter may provide.

Were we to approach Euler's problem of classification today, we might first write the governing equations in the intrinsic form

$$
\begin{aligned}
\left(\Re \theta^{\prime}\right)^{\prime}+\lambda \sin \theta=0, & x^{\prime}=\cos \theta, \quad y^{\prime}=\sin \theta, \\
\lambda=\text { resultant force, } \theta= & \begin{array}{l}
\text { slope of the tangent measured } \\
\text { from the axis of the resultant } \\
\text { force. }
\end{array}
\end{aligned}
$$

If $\mathscr{B}=$ const., then phase-plane methods suffice to analyse the differential equation and determine the qualitative properties of solutions. Euler approached the differential system with bare hands.

Euler's analysis divides the possible forms into nine classes. The ninth is the circle; the first is a straight line or a small perturbation of it, which Euler shows to be a sine wave of small amplitude. Figure 3 reproduces Euler's sketches for the other seven classes. The fourth class is really three: one for curves like that drawn, one consisting in the curve whose humps are tangent to one another, and one for curves whose successive humps intersect at two points. Euler calculates very accurate numerical values for some special and important points, but otherwise numerical work plays no part. The curves are not plotted; they are sketched on the basis of rigorous arguments concerning the slope as a function of $s$ or $x$, bounds obtained as necessary and sufficient for the integrands to be real, the number and nature of points of inflection, periodicity and the failure thereof. It is a qualitative analysis of the two integrals. Both are general elliptic integrals of the first kind; Euler's theory is complete. The nature of the corresponding elliptic functions can be read off from his graphs by looking at $x$ as a function of $y$. So far as I know, the next example of qualitative analysis comes just 150 years later, in Poincaré's Celestial mechanics. Still later, Born, in his thesis of 1906, was the first to publish figures of elasticas plotted numerically and to check the theory against experiments on springy wires.

A traditional date for the birth of elliptic functions is 23 December 1751, on which Euler was asked to review Fagnano's collected works, which results first published as early as 1714. Euler had been studying elliptic integrals and elliptic functions since 1738, when he wrote to the Bernoullis that he had "noticed a singular property of the rectangular elastica" having unit excursion:

$$
\text { length } \times \text { height }=\int_{0}^{1} \frac{d x}{\sqrt{1-x^{4}}} \cdot \int_{0}^{1} \frac{x^{2} d x}{\sqrt{1-x^{4}}}=\frac{1}{4} \pi .
$$

This property and his analysis of elastic curves joined Fagnano's geometrical investigation of lemniscate functions in providing the basis for Euler's work 


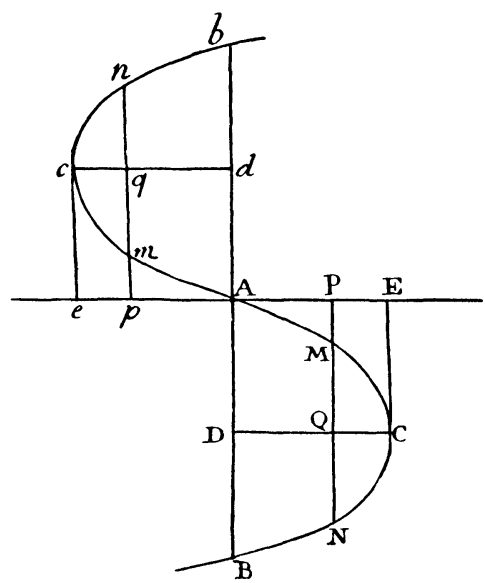

3a. Class 2

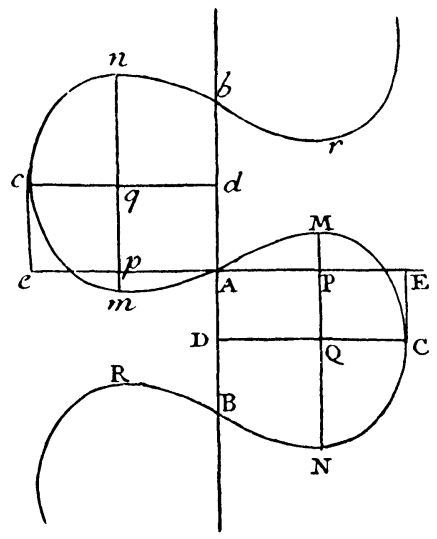

3b. Class 4

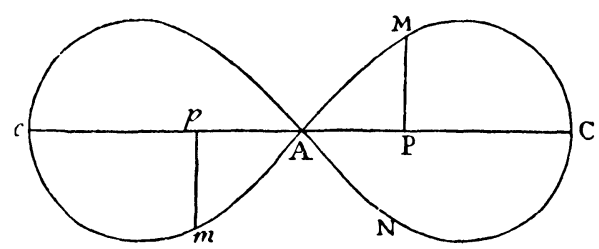

3c. Class 5

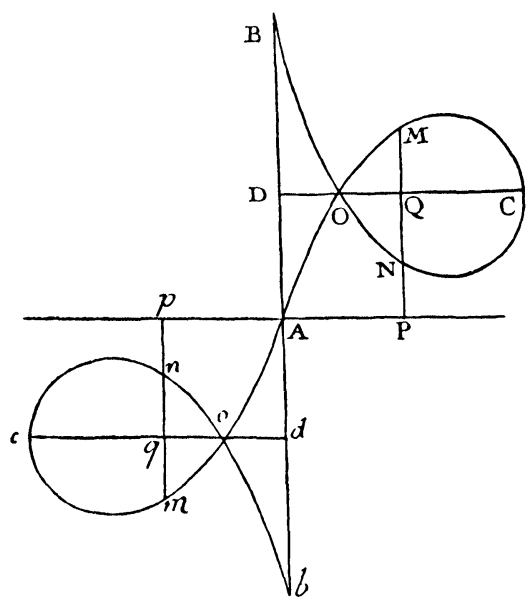

3d. Class 6

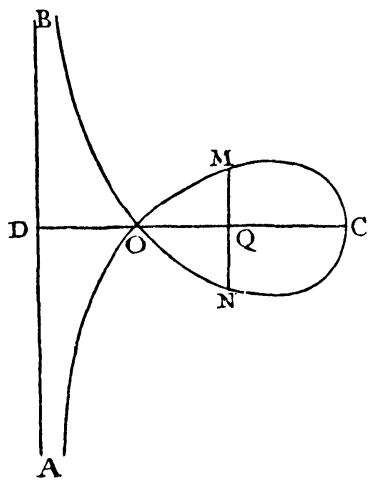

3e. Class 7

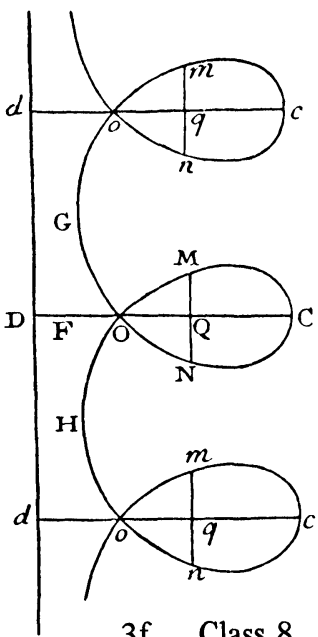

3f. Class 8

FIGURE 3. Euler's sketches for various classes of elasticas 
toward his discovery of the addition theorems for elliptic functions in the 1770s. The nineteenth and twentieth centuries' appeal to the theory of elliptic functions for treating problems like that of the elastica was not an unmixed blessing for mechanics or for mathematics more generally. It suppressed Euler's direct, nonlinear thinking in favor of properties of special functions, which came to be ends of research rather than means to solve a natural problem.

In the theory of the elastica the straight, unbent form is always possible. Thus a bent form is always a second and nontrivially different solution for an elastica subject to opposing collinear loads. Euler's figures make it obvious that each shape having a point of inflection gives rise to infinitely many such solutions (Figure 4) for each load $P$. If for given $\mathscr{B}$ the pair $P,-P$ will equilibrate bent forms of length $l$, it will also equilibrate bent forms of lengths $2 l, 3 l, \ldots$, respectively, having $2,3, \ldots$ bays, respectively. Not only that, Euler saw at once that a bent form is not possible unless the load $P$ was large enough:

$$
P>P_{C}, \quad \text { “critical load" } P_{C}=\pi^{2} \frac{\mathscr{B}}{l^{2}}
$$

Here we see the essential role of the material properties expressed by the parameter $\Re$. One reinterpretation of the preceding statement about the bays is that for a bar of given length $l$, the loads $4 P_{C}, 9 P_{C}, \ldots$ must be exceeded in order for the bent forms with $2,3, \ldots$ bays to become possible. A single problem has $n$ nontrivially distinct solutions if the ratio $P / P_{C}$ lies in the interval $\left[(n-1)^{2}, n^{2}\right]$. Thus begins the problem of bifurcation, which was not to be touched again, so far as I know, until Poincaré investigated rotating figures of equilibrium.
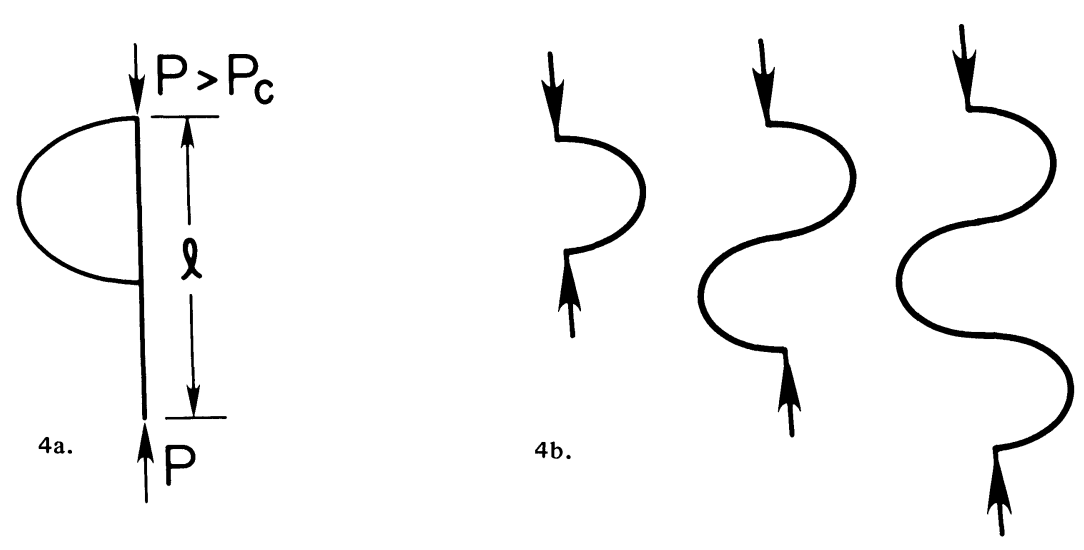

Figure 4a. The unbent and simplest bent forms for an elastica of length $l$ subject to thrust greater than critical.

FIGURE $4 \mathrm{~b}$. Three of the infinitely many bent elasticas corresponding to a given thrust greater than critical. 
Euler noticed also that if the nonlinear differential system is approximated by a single linear equation supposedly valid for nearly straight solutions, the critical load thereby determined is the same as for the exact theory. The sequence of exact critical values of $P$ is just the same as the sequence of proper numbers of the corresponding linearized problem. Nevertheless, according to the linearized theory the amplitude of the bent form is arbitrary. Here again is the first instance of a familiar property of problems of bifurcation.

Fourteen years after he wrote his great treatise Euler attacked the same class of problems for bars of nonuniform section, $\mathscr{B}=f(s)$. In the latter nineteenth century, when Latin was less widely understood and linearity was regarded as a fact of physics, this later paper in French was taken as the source of Euler's theory. The magnificent clarity of Euler's work of 1743 was thereby lost. Until fifty years ago engineers in attempting to remove the defects of the linear theory sometimes rediscovered approximations to Euler's exact results, sometimes instead published erroneous formulae designed to be such.

All this work on the elastica presumes it to be a plane curve. Euler investigated also the skew elastica. Just as to solve the problem of the catenary required determination of the curvature, the skewing of a rod required the concepts of osculating plane and binormal. Early in the eighteenth century John Bernoulli had described the osculating plane in words but had not developed the specific quantities and relations associated with it. Euler provided those in a paper of 1774 and developed them more clearly in a paper presented in the following year. One week later he read a purely geometrical paper that goes so far as to obtain the first of the "Serret-Frenet" formulae: In modern notation, $\mathbf{t}^{\prime}=\boldsymbol{\kappa} \mathbf{n}, \mathbf{n}$ being the principal normal (introduced by Euler in his book on rigid bodies, 1765). The second "Serret-Frenet" formula, namely $\mathbf{b}^{\prime}=-\tau \mathbf{n}$, was to be found in 1826 by another of the great creators of elasticity, Cauchy.

3. Systems in small vibration: proper numbers and simple modes, linear differential equations, "Bessel" functions, "Laguerre" polynomials, integral transforms, resonant oscillations. Mechanics, as it came down from the seventeenth century, did not suffice to set up differential equations of motion for systems of any complexity. Partial differential equations did not begin to appear until nearly the middle of the eighteenth century. In the heroic period before that time many great problems regarding small oscillations were set by appeal to a special principle introduced by Taylor in 1713: The acceleration is proportional to the displacement from equilibrium. Equivalently, each particle of the system oscillates like a simple pendulum, and all particles oscillate at the same frequency. The modern reader sees at once from the equations of motion that for a linear system this assumption limits the solutions to the simple modes, determined by an ordinary differential system for a vector $\mathbf{y}$ :

$$
\mathbf{L y}=-\omega \mathbf{y},
$$

in which $\mathbf{L}$ is a linear differential operator and $\omega$ is the frequency proper to the mode. The differential equation and suitable end conditions or conditions at $\infty$ determine $\omega^{2}$ uniquely and determine the mode to within an arbitrary constant. Taylor was able to extract no more from this method than the frequency and sinusoidal shape of the fundamental mode of the vibrating 
string, but Daniel Bernoulli in 1733 got major results. The first two of the several systems successfully treated by him and by Euler are the massless cord loaded by $n$ weights and the continuous heavy cord (Figure 5). The solutions are expressed in terms of what are now called "Laguerre polynomials" and

Comment:Acad:Sc.Tom:VI.Tab:VII.p.108.
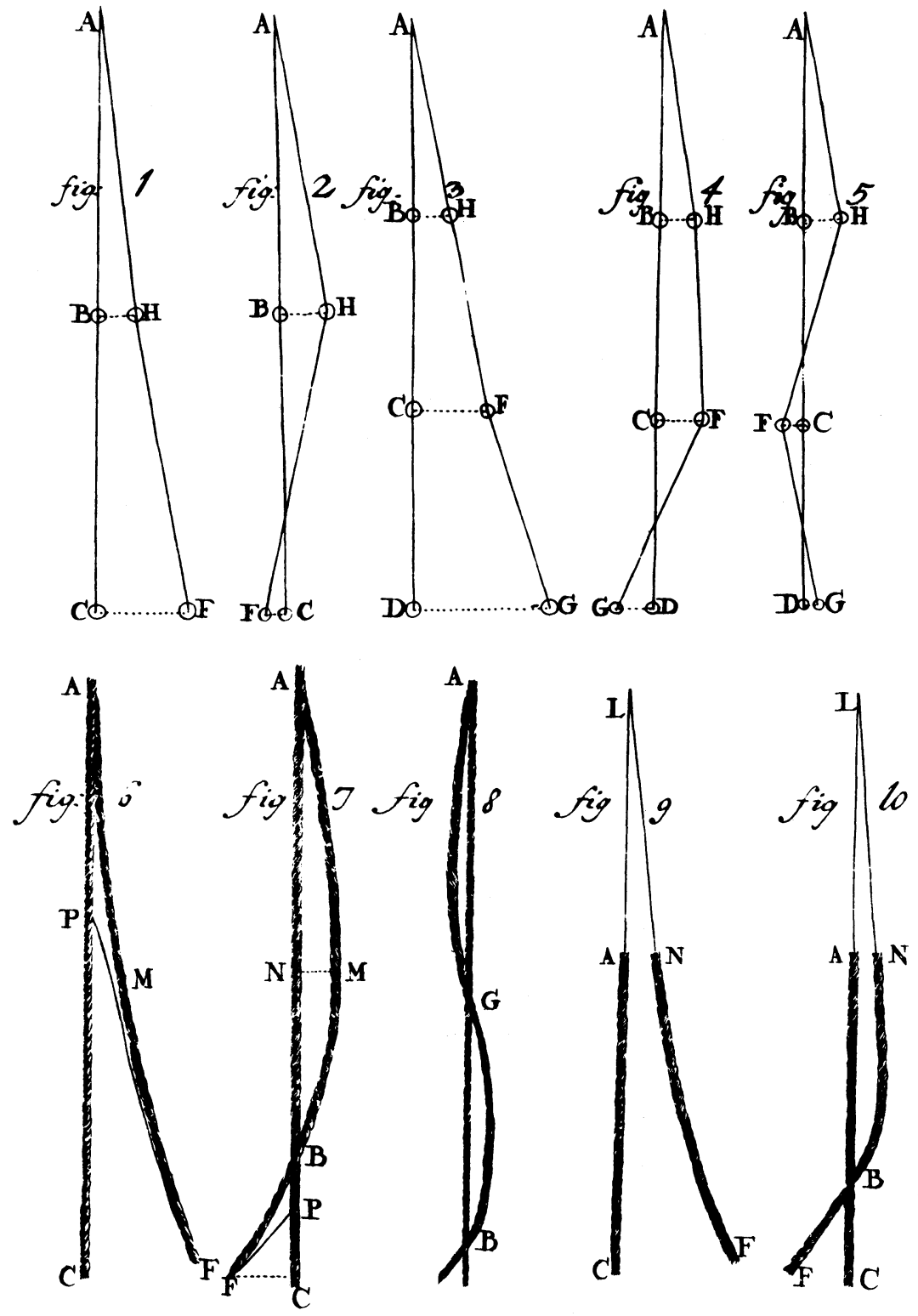

Figure 5. Daniel Bernoulli's figure to illustrate all the modes and nodes possible for bifilar and trifilar pendulums and the modes of lowest order for two kinds of continuous pendulums. 
"Bessel functions", respectively. Euler introduced integral transforms in connection with the latter in 1735 .

In that same year both Euler and Bernoulli faced the apparently more difficult problem of transverse vibrations of an elastic rod. They both arrived at the differential equation

$$
d^{4} y / d x^{4} \propto y .
$$

Neither could solve it at first except in infinite series. These are two great mathematicians. Please recall that the year is 1735 ! Nothing becomes obvious until somebody first sees it. By 1739 both Euler and Bernoulli had seen how to integrate the differential equation

$$
\sum_{k=0}^{n} A_{k} \frac{d^{k} y}{d x^{k}}=0, \quad A_{k}=\text { const. }
$$

including the modifications necessary when the characteristic equation has repeated roots. Until this time only particular differential equations had been studied. Here begins the general theory.

In the same year Euler was shown an experiment in which a watch was set in motion by being used as the bob of a pendulum of suitable length. He at once set up and solved the differential equation of harmonically forced oscillation:

$$
M \ddot{x}+K x=F \sin \omega_{0} t .
$$

He showed that when the natural frequency $\sqrt{K / M}$ equalled the driving frequency $\omega_{0}$, the amplitude tended to $\infty$. Here begins the mathematical theory of resonant systems.

For vibrations of all these systems having $n$ or infinitely many degrees of freedom, Daniel Bernoulli had observed that there are $n$ distinct proper frequencies and that the $k$ th mode has $k-1$ nodes. If $n=\infty$, the same statement holds. Of course everyone saw that a linear combination of simple modes also provided a solution; that is, the simple modes may be excited separately, and if excited simultaneously they do not interfere with each other. Mathematical analysis in the eighteenth century did not go beyond these statements, for nobody exploited the orthogonality of the trigonometric functions or perceived the similar relations satisfied by the functions appropriate to other simple modes.

4. Partial differential equations: the concept of "solution", discontinuities, the "modern" definition of function, functional basis, singularities, generalized solutions. In the years 1742-1748, at last, John Bernoulli, D'Alembert, and Euler obtained differential equations of motion for oscillatory systems of finitely or infinitely many degrees of freedom. Before that, Euler had obtained and studied a partial differential equation for a geometrical problem, but the first partial differential equation of motion was D'Alembert's for the heavy hanging cord. In 1746 he obtained the counterpart for the string in infinitesimal transverse vibrations:

$$
\frac{1}{c^{2}} \frac{\partial^{2} y}{\partial t^{2}}=\frac{\partial^{2} y}{\partial x^{2}}, \quad c=\text { const. }
$$


He at once perceived the solution

$$
y=f(x-c t)+g(x+c t),
$$

where $f$ and $g$ are arbitrary "equations". D'Alembert was never able to explain precisely what he meant by an "equation". His remarks are correct for analytic functions, but some of his examples are not analytic. For him a triangle was not given by an "equation" because the "equations" for the two legs are different. Sympathetic modern readers have uttered the term "analytic continuation" to help reconcile themselves with what D'Alembert wrote. For D'Alembert the vibrating string had to satisfy the partial differential equation at the endpoints of its interval of definition (limits from outside as well!). The effect of his restrictions was to limit solutions to the simple modes; he declared that if the initial conditions were not given by "equations", the solution was "impossible".

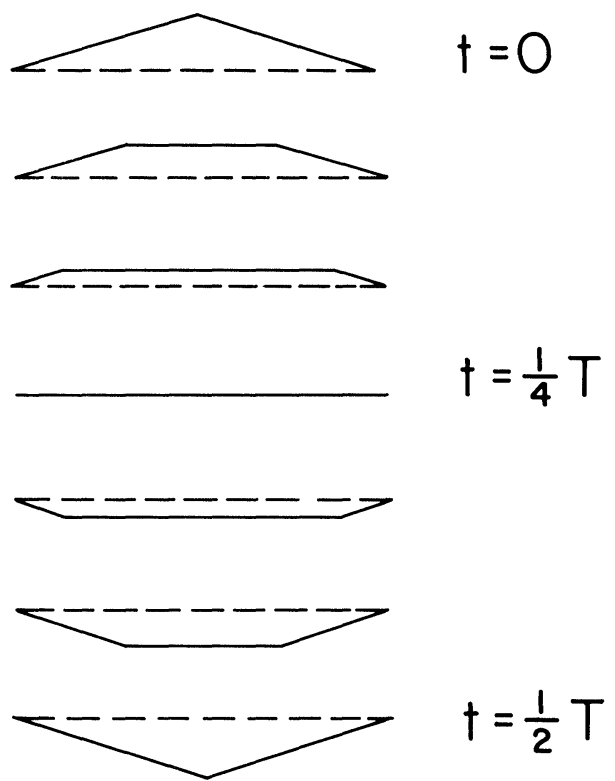

FIGURE 6. Sketch adapted from Euler's figure of 1772 to show shapes assumed by the string plucked into a triangle and then released.

Euler refused to let a mathematical prejudice render any physically natural problem "impossible". He saw that the concepts "solution" and "function" both needed to be broadened. He considered functions whose graphs were "freely drawn by the hand". The triangle as an initial form had been discussed by musicians for a century. While Euler did not exhibit his solution for the triangular form until 1772, it is contained in his general solution of 1748, elaborated in 1754 and 1764-1765. Figure 6, adapted from a sketch in Euler's 
paper of 1772, shows that he considered only the interval of definition, nothing outside it; that in the concept of "solution" he not only excused the endpoints but also allowed a finite number of interior points at which the slope and the velocity did not exist; that the effect of the endpoints is to create and destroy moving singularities; and that his solution is explicit and exact by modern standards. Euler constructs the solution for arbitrary initial conditions by drawing specified extensions of the initial shape and curve of initial velocity to $\infty$ and $-\infty$, then sliding these across to right and left and adding the ordinates. Thus his solution is effective numerically.

It was in this context that Euler was led to his long neglected but now famous second definition of a function (1755):

If $\ldots x$ denotes a variable quantity, then all quantities which depend upon $x$ in any way or are determined by it are called functions of it.

He spoke also of "every method by which one quantity could be determined by others". His explanations indicate that he excluded constant functions, but otherwise his definition is not so restrictive as Lobatchevsky's (1834) and Dirichlet's (1837), which refer only to continuous functions.

While the problem of the vibrating string leads to solutions with discontinuous derivatives, it requires the solution itself to be continuous. The interpretation of the linear wave equation in terms of the vibrations of a column of air in a flute, on the contrary, makes discontinuous solutions reasonable. A striking example is the function whose value is 0 at all points but one (Figure 7). Euler applied his solution to this function as an initial shape and another such function as an initial speed. He obtained explicit rules for the propagation of such pulses and their reflection from an open end or closed one. His rules for pulses illustrate the later "method of images". Since such discontinuous functions provide a basis for all functions, all solutions may be obtained by combining solutions for pulses.

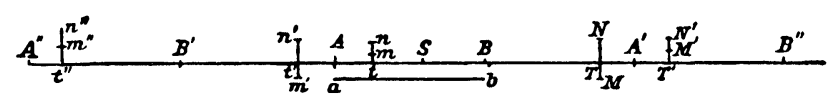

FIGURE 7. Euler's diagram to explain his solution representing the propagation and reflection of pulses of width 0 in the string of length $\mathrm{AB}$.

Euler attempted to justify in various ways his regarding discontinuous functions as "solutions". In effect, he discarded the partial differential equation altogether and took the sum of arbitrary functions as being the general statement of the physical problem; continuity and differentiability play no part in a statement of this kind. He observed that a solution with discontinuous slope could be regarded as the limit of a family of smooth solutions, for which his construction was necessary as well as sufficient for solution. Finally, in 1760, when treating a string with discontinuous density, he replaced the condition of continuous slope, which cannot be satisfied, by an integrated 
expression equivalent to it for differentiable solutions. These three examples join Euler's famous definition of the sum of a divergent series to show his approach to problems arising from irregularity. Some of his attempts are almost "modern" in spirit. Concepts of weak and distributional solutions can be traced back to him.

What about the "Fourier series" for solving the problem of the vibrating string and others like it? Daniel Bernoulli always thought that the most general motion of such a system could be expressed as the sum of a series of suitably selected simple modes, and in 1753 he came out with a general statement to this effect, which he regarded as "a new truth of mathematical physics". For Euler, such statements were true if and only if they could be derived from equations of motion. He succeeded in providing such justification, more or less, for a system of $n$ coupled harmonic oscillators, but not more generally. Although in the eighteenth century particular examples were exhibited, no mathematical theorems on expansions in series of proper functions were proved. Not much more can be said for Fourier in his celebrated treatise of 1822. He there published a ridiculously false proof for his claim that all functions could be expanded in a trigonometric series, which is untrue, and he treated such series as if they determined the functions to which they corresponded, which also is untrue. Euler, to illustrate his solution of the wave equation and to provide a basis for all solutions, used a pulse; Fourier's method makes that function indistinguishable from a constant function and so nothing can come of it for solving differential equations.

Justification of Bernoulli's great idea had to wait for more scrupulous analysts of a period later than Fourier's.

5. Plates: the "Gaussian" curvature. The Paris prize of 1811 required a theory of vibrations of elastic plates. The only entrant was S. Germain; her proposal and analysis were totally incorrect, but she did consider the possibility that the forces required to bend an elastic plate might depend upon its principal curvatures $\kappa$ and $\kappa^{\prime}$ through the combinations $\kappa+\kappa^{\prime}$ and $\kappa \kappa^{\prime}$, both of which had appeared in researches on the differential geometry of surfaces. Poisson succeeded in proving the surface integral of $\kappa \kappa^{\prime}$ to be determined by boundary values alone. In 1814 he asked Rodrigues, a student twenty years old, to look into the matter. In a note published in 1815 Rodrigues introduced the auxiliary sphere and proved the value of the surface integral of $\kappa \kappa^{\prime}$ (Gauss' "curvatura integra", total curvature) to be equal to the area of the normal projection of the surface onto that sphere. This statement is the first step toward the Gauss-Bonnet theorem. Rodrigues' article contains almost exactly, but in reverse order, what Gauss was to publish more than a decade later in the first six sections of his Disquisitiones. The exception: Rodrigues noticed that the map of a closed surface onto the sphere may cover it more than once. For example, while the total curvature of an ellipsoid is $4 \pi$, Rodrigues gives the total curvature of a torus as $8 \pi$. Although Rodrigues fails to notice the change of sign that makes the correct total curvature of a torus 0 , his considerations here provide the earliest example of differential geometry in the large. 
6. The three-dimensional theory: deformation of regions, strain and local rotation, tensors, proper numbers and proper vectors, polar decomposition, spectral resolution, isotropy and hemitropy groups. The principal creator of three-dimensional elasticity is Cauchy. Mainly for use in three-dimensional hydrodynamics, Euler had introduced general mappings of regions and had created the associated calculus of partial derivatives, chain rules, Jacobian determinants, etc.; he had also formulated the general principles of linear and rotational momentum and had shown how to apply them to fluids. Cauchy mastered all this and turned it to use in elasticity. Tensors had not yet been isolated as a class, but examples were already familiar. Using geometrical representations by quadric surfaces, Cauchy discovered and proved the "polar" decomposition: Any invertible tensor can be expressed as the product of a pure dilatation by a rotation, in either order, the rotation being the same for each (Figure 8). He derived the laws of transformation relating components with respect to different rectangular co-ordinate systems. He demonstrated the vectorial character of the "curl" of a vector and established its meaning as an approximation to an orthogonal tensor. He discovered and proved the spectral decomposition theorem for symmetric tensors. He introduced also the principal invariants of a tensor: the sums of its latent roots taken 1,2, and 3 at a time. The number 3 here reminds me that nearly all this material generalizes easily to $n$ dimensions. It is fair to say that much of the algebra of vectors, matrices and tensors grew from Cauchy's work on the strain, local rotation, and stress in elastic bodies. Even the notation with kernel index and subscripts, which became common later in Riemannian geometry, first appeared in relation to Cauchy's famous stress tensor, was adopted by him, and with subscripts 1, 2, 3 was introduced by Clebsch in his treatise on elasticity (1862).
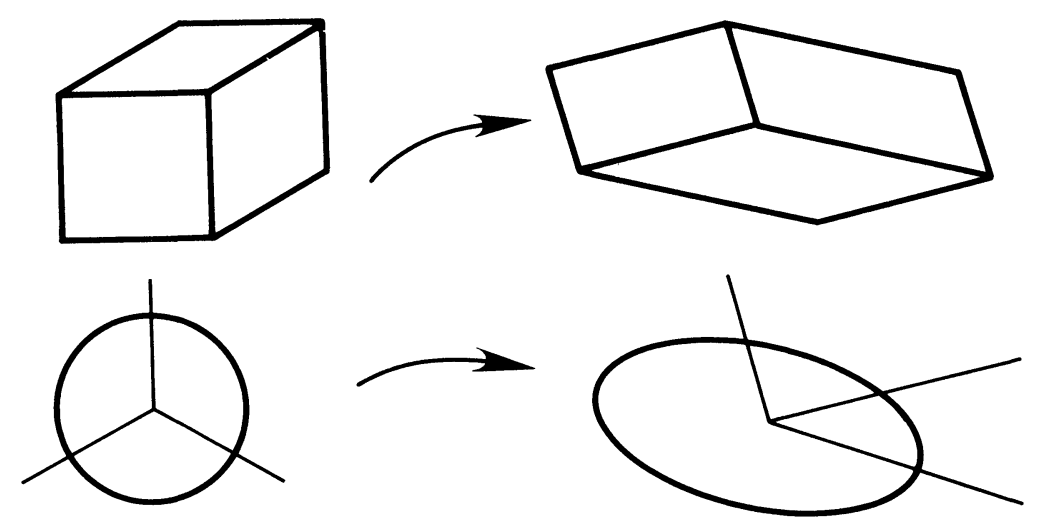

FIGURE 8. Sketch to illustrate Cauchy's polar decomposition theorem. 
Most of the terms I have used are modern. For example, "tensor" was introduced by Hamilton in his translation of some of Cauchy's theorems into the formalism of quaternions. Its name reflects its nature: It extends vectors as well as turning them. Quaternions themselves were first discovered, applied, and published by Rodrigues, Poisson's former pupil, in 1840.

To Cauchy we also credit the concept of functions invariant under rotations. Such functions for the full orthogonal group are called isotropic; for the proper orthogonal group, hemitropic. The former term was introduced by Cauchy, but in the sense of the latter. He discovered and established, for an isotropic linear function $\mathbf{f}$ mapping the space of symmetric tensors into itself, the representation

$$
\mathbf{f}(\mathbf{S})=\alpha(\operatorname{trace} \mathbf{S}) \mathbf{1}+\beta \mathbf{S} .
$$

Another theorem of his states that an isotropic scalar function of a symmetric tensor equals a function of the principal invariants of that tensor. Another: $A$ hemitropic function of three-dimensional vectors equals a function of the inner products and triple products that may be formed from them.

While most of Cauchy's discoveries that I have mentioned grew from his elasticity of continuous bodies, the last was suggested by his theory of the stress in a crystal lattice. In this connection he considered invariance under subgroups of the orthogonal group. The problems Cauchy encountered in elasticity and the concepts he introduced and developed there provided one of the motivations for his celebrated memoir on group theory, in which we first encounter the concept of abstract group.
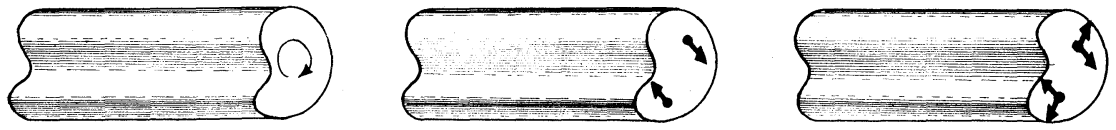

FIGURE 9. Three equipollent torsional loads on a cylinder.

7. Torsion of a cylinder: principal solution of a system of differential equations and its status as an approximation to other solutions. Under fairly weak assumptions it can be shown that the solutions of standard boundaryvalue problems of linear elasticity exist and are unique, at least to within a rigid displacement of the body as a whole. Such is the case for torsion: A cylinder left free on its mantle is twisted by tractions applied to its ends (Figure 9). The resultant force of the applied tractions is naught; the resultant torque is directed along the generators. A generalized "Neumann" problem results: A solution of an elliptic system of linear partial differential equations with constant coefficients is sought subject to the condition that on the boundary certain linear combinations of partial derivatives equal prescribed functions of place. Any two such solutions differ by at most an infinitesimal rotation. In other words, different boundary values give rise to nontrivially different solutions. The differential equations to be satisfied by the displacement $\mathbf{u}$ have the vectorial expression

$$
\Delta \mathbf{u}+\frac{1}{1-\nu} \operatorname{grad} \operatorname{div} \mathbf{u}=0, \quad \nu=\text { const. }<\frac{1}{2} .
$$


If $2 \mathbf{E} \equiv \operatorname{grad} \mathbf{u}+(\operatorname{grad} \mathbf{u})^{T}$, the traction vector $\mathbf{t}$ at a point of the boundary where the outer unit normal is $\mathbf{n}$ is given by

$$
\frac{\mathbf{t}}{\mu}=2 \mathbf{E n}+\frac{\nu}{1-2 \nu}(\operatorname{trace} \mathbf{E}) \mathbf{1}, \quad \mu=\text { const. }>0 .
$$

The mantle of the cylinder is free of traction: $\mathbf{t}=\mathbf{0}$. The plane ends, which we may denote by $e^{ \pm}$, are subject to traction fields $\mathbf{t}$ consistent with constraints written as follows in terms of integrals over $e^{ \pm}$:

$$
\int_{\mathcal{C}^{ \pm}} \mathbf{t} d A=\mathbf{0}
$$

$$
\begin{gathered}
\mathbf{i} \cdot \int_{\mathcal{C}^{ \pm}}(\mathbf{p}-z \mathbf{k}) \times \mathbf{t} d A=\mathbf{j} \cdot \int_{\mathcal{C}^{ \pm}}(\mathbf{p}-z \mathbf{k}) \times \mathbf{t} d A=0, \\
\mathbf{k} \cdot \int_{\mathcal{C}^{+}}(\mathbf{p}-z \mathbf{k}) \times \mathbf{t} d A=-\mathbf{k} \cdot \int_{\mathcal{C}^{-}}(\mathbf{p}-z \mathbf{k}) \times \mathbf{t} d A=M,
\end{gathered}
$$

in which $M$ denotes the torque applied and hence is a given constant, while the origin of the Cartesian co-ordinates $x, y, z$ is the centroid of the central cross-section $\Theta, \mathbf{p}$ is the position vector $\mathbf{p}=x \mathbf{i}+y \mathbf{j}+z \mathbf{k}$, the axes of $x$ and $y$ are principal axes of inertia of $\mathcal{C}$, and $z=h$ on $\mathrm{C}^{-}, z=-h$ on $\mathrm{C}^{+}$.

Nevertheless, the problem that nature presents us is not described well by this mathematical formulation. When we twist a shaft, we do not know what tractions we apply to its ends. We grasp the ends of the shaft in some way, say with a pipe-wrench, and we apply a torque. Alternatively, we may build the shaft into a framework and ask how much it is twisted by the action of other members joined to its ends. We ask how much a shaft of given cross-section and material twists in response to the torque applied. We know that the practical answer depends upon the material, say steel or rubber, and upon the cross-section, but usually no more than that. The answer according to the theory depends upon much more. To determine the solution, in principle we must also know the way the twisting forces are distributed over the cross-section. As we see from (A), the torque $M$ is determined by integrating those forces. In practice only that integral, not the quantities being integrated, can be determined. An infinite class of boundary-value problems corresponds to a single physical problem. Not only, usually, can we not in applications to real bodies determine conditions sufficient to get a unique solution in the mathematical theory, but usually we do not wish to determine them. The three-dimensional theory is here too refined for its intended use. We must somehow cut away the superfluous detail and retain only the essentials. Indeed, Coulomb's experiments in the late eighteenth century had suggested that

$$
\tau_{m}=M / \mu R,
$$

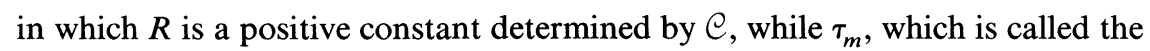
overall twist, is the quotient by $2 h$ of the angle of relative rotation of the two ends. The constant $\mu R$ is the overall torsional rigidity of the cylinder. The mechanical engineer can consult a handbook to find $R$ for various commonly used cross-sections. The answers he finds there refer to $\mathcal{C}$ alone and make no mention of $h$. 
The practical rule (C) is a good one, and the theory of elasticity should substantiate it. These facts were recognized, though not expressed in this way, by Barré de St. Venant in his great memoir on torsion, presented in 1853 . He saw how to determine a single, particular solution corresponding to each torque. To that end he chose to consider a particular class of putative solutions:

$$
\mathbf{u} / \tau=-y z \mathbf{i}+x z \mathbf{j}+\Phi(x, y) \mathbf{k},
$$

in which $\tau$ is an arbitrary constant and $\Phi$ is a function to be determined. All pairs of transverse sections of the cylinder experience the same overall twist $\tau_{m}$, and $\tau_{m}=\tau$; thus $\tau$ deserves to be named the twist with no qualification. All transverse sections are congruent and parallel surfaces having the shape $z=\Phi(x, y)+$ const. St. Venant showed that such solutions exist and are essentially unique in the specified class; that the warping function $\Phi$ is determined to within a constant by $\mathcal{C}$ through solution of a problem of potential theory in the plane; that the conditions (A) are satisfied; and that (C) holds if and only if the torsional rigidity $R$ is determined from $\Phi$ through the formula

$$
R=\int_{\mathfrak{C}}\left[\left(\frac{\partial \Phi}{\partial x}-y\right)^{2}+\left(\frac{\partial \Phi}{\partial y}+x\right)^{2}\right] d A,
$$

which, like $\tau$, is independent of $h$. Values of $R$ gotten through this formula have been found good, and when you look up the torsional rigidity of a particular shape in an engineer's handbook, usually it is this mathematical quantity that you find listed. For more than a century mathematicians have devoted much effort to calculating and estimating it for a general cross-section. Thus St. Venant, besides solving the major problem of nineteenth-century elasticity he had set himself, provided a field of study for analysts of the twentieth century, but that is not the aspect of analysis I wish to emphasize here.

Rather, I refer to the relation between the particular representative found by St. Venant, which we may call the principal solution of the torsion problem, and the infinitely many other solutions that correspond to the same problem when it is approached through the general formulation of classical elasticity. St. Venant expressed his convictions as follows:

...The manner in which the forces are applied and distributed towards the extremities of a prism is indifferent to the sensible effects produced upon the rest of its length, and so always we may with sufficient approximation replace the forces actually applied by statically equivalent forces....

Hence, the principal solution serves through much of the body as a sufficient approximation to all the solutions in its class.

The vague statement I have just quoted and rephrased is called St. Venant's Principle; so are various generalizations and variants of it. Much effort has been put out on St. Venant's Principle, especially in the last forty years. Sometimes we forget that before proceeding to prove a theorem, usually we must formulate it. In mathematics today, dominated as it is by clever technique 
and assiduous technicians, too little attention is paid to conjecture, formulation, and concept. There are many different precise statements that seem to correspond to St. Venant's assertion or extend it. Several of these have been formulated; some have been proved false, some true, and some remain uncertain; new formulations are put forward every few years.

I will not describe any of this work. My purpose is to show that elasticity gave rise to ideas on how to dispose of, yet make precise use of, data too abundant to be useful even if it could be determined in practical application. The first example is provided by James Bernoulli's elastica, which for mathematical theory replaces a beam by a line having some properties associated with a bent body. The second, which came after Cauchy's general theory, not before it, is St. Venant's treatment of torsion, in which he obtains within the possibilities of the general theory a particular and especially simple solution from which major properties of an infinite class of solutions may be determined.

Most mathematicians have not heard of St. Venant's Principle. Many are familiar with Prandtl's idea of a viscous boundary layer, which claims status for a peculiar "approximation" and points to status for a corresponding flow of an ideal fluid. Something similar, but referring to approximation for small thickness rather than for a small value of a material parameter, arose earlier, but in a less fuzzy way, in Kirchhoff's attempt to relate the theory of thin elastic plates to the general theory of elasticity in three dimensions. St. Venant's Principle should be simpler in that it compares solutions according to one and the same theory. It distinguishes one particularly simple, particularly smooth, and particularly important solution and claims for it a status with respect to all solutions. Nevertheless, its proper formulation remains in debate.

I am sorry to have to remark that many analysts accept as a "derivation" of a basic equation some remarks and mere assertions of the "physical" or "engineering" kind, to which they give a total credence they would reject with contempt were they to enounter it in a mathematical argument. My foregoing remarks do not refer to heuristic "derivations" but to strict, mathematical comparison of the respective sets of solutions of two different equations, one of which is designed (for whatever reason) to approximate the other. The question is, in what sense does a particular solution of the "approximate" equations in fact approximate members of the corresponding class of solutions of the equations being "approximated"? For the general problem of torsion the basic differential equations are $(\mathrm{N})$ with the boundary conditions (A); for a fixed contour $C$ and fixed constants $h$ and $M$, this problem has infinitely many solutions. The "approximate" differential equations are $(\mathrm{N})$ reduced by substitution of St. Venant's hypothesis (StV). For fixed $\mathcal{C}$ and $M$ there is one and only one St. Venant solution. It is a member of the infinite class of solutions of the general problem of torsion. What is its status with respect to that class?

A problem of the same kind, but much more difficult both in principle and in analysis, arose in the kinetic theory of gases. There it is a question of evolution in time, perhaps easier to conceive, but harder to handle because the kinetic theory rests on a nonlinear integro-differential equation for which there is not yet even a basic, general theory of existence and uniqueness. 
The recent investigations of these fundamental issues of mechanics may lead to a new branch of analysis: the theory of principal solutions of differentialfunctional equations.

Just in the last few years Muncaster has formulated and studied a general, abstract structure for pairs of theories, a "fine" one laid down as "exact" and general, and a "coarse" one designed to deliver with less expense of analysis and calculation the essential or overall features of a class of solutions of the "fine" theory. In some instances the solution of a problem in the coarse theory turns out to be a principal solution of a corresponding problem in the fine theory.

For the history of elasticity in generai:

\section{REFERENCES}

A.-J.-C.-Barré de St. Venant, Historique abrégé des recherches sur la résistance et sur l'élasticité des corps solides, pp. xc-ccexj of C.-L.-M.-H. Navier, Resumé des Leçons... sur l'Application de la Mécanique..., Paris, Dunod, 1864.

I. Todhunder, A history of the theory of elasticity... (edited and completed by K. Pearson), Cambridge Univ. Press, Vol. 1, 1886, Vol. 2, 1893. Both of these histories are far from complete.

For details of $\S \S 1-4$ :

C. Truesdell, The rational mechanics of flexible or elastic bodies, 1638-1788, Leonhardi Euleri Opera Omnia, Ser. II, Vol. 11, part 2, Orell Füssli, Zürich, 1960.

For §5:

C. Truesdell, Sophie Germain, Arch. Hist. Exact Sci. (in preparation).

For the work of Cauchy there is no general survey. The following four works contain hundreds of references to specific researches of his on elasticity, tensor fields, etc. Help in finding them in the first three is provided by the lists of works cited; in the fourth, by the index of authors cited.

C. Truesdell and R. Toupin, The classical field theories, Encyclopedia of Physics (Flügge, ed.) Vol. III/1, Springer-Verlag, Berlin, 1960, pp. 226-793.

J. L. Ericksen, Appendix [to the preceding], Tensor fields, op.cit., pp. 794-858.

C. Truesdell and W. Noll, The nonlinear field theories of mechanics, op.cit., Vol. III/3, 1965.

C. Truesdell, Essays in the history of mechanics, Springer-Verlag, New York, 1968.

For the basic mathematics of linear elasticity, including St. Venant's Principle:

M. E. Gurtin, The linear theory of elasticity, Encyclopedia of Physics (C. Truesdell, ed.), Vol. VIa/2, Springer-Verlag, Berlin, 1972, pp. 1-295.

Mainly for nonlinear elasticity:

J. E. Marsden and T. J. R. Hughes, Mathematical foundations of elasticity, Prentice-Hall, Englewood Cliffs, N.J., 1983.

C.-C. Wang and C. Truesdell, Introduction to rational elasticity, Noordhoff, Leyden, 1973.

For principal solutions:

C. Truesdell and R. G. Muncaster, Fundamentals of Maxwell's kinetic theory of a monatomic gas treated as a branch of rational mechanics, Academic Press, New York, 1980. See Chaps. XIV, XV, and Epilogue.

R. G. Muncaster, Invariant manifolds in mechanics: The general construction of coarse theories from fine theories, Arch. Rational Mech. Anal. (to appear).

General note:

Many mathematical researches on St. Venant's problem, nonlinearly elastic rods, and other aspects of elasticity, may be found in Archive for Rational Mechanics and Analysis, 1957 onward. Of these, many contain specific references to the work of Cauchy, Euler, St. Venant, and other authors mentioned in the above lecture.

The Johns Hopkins University, Latrobe 119, Baltimore, Maryland 21218 